\title{
Parametric uncertainty bounds for performance robustness of linear systems with output feedback
}

\author{
C. Wen \\ I-K.Fong
}

Indexing terms: Performance robustness analysis, Parametric uncertainty bounds, Linear systems

\begin{abstract}
The paper is devoted to performance robustness analysis of uncertain state-space models with linear parametric uncertainties and output feedback. The purpose is to find bounds on uncertain parameters within which the system $\mathrm{H}_{2}$ norm performance index is kept below a prespecified value. Through matrix nonsingularity analysis, it is shown that the bounds can be computed from structured singular values of certain composite matrices. To fully utilise the structural information of uncertainties, the authors also develop a method for reducing matrix sizes involved in the computation of structured singular value.
\end{abstract}

\section{Introduction}

Over the years, the precise and fixed linear control schemes have been used extensively in many engineering applications. While the real system behaviour is often described by mathematical formulation, it is almost impossible to get an exact model for the system due to the existence of various uncertainties. Here, we focus on systems with parametric uncertainties. To be more specific, we consider linear state-space models with uncertain parameters added to system, input, and output matrices in a linear fashion. Also, we assume that a stabilising output feedback controller is designed for the nominal system, hence the closed-loop state equations have quadratically coupled parametric uncertainties in the system matrix. Stability robustness analysis of this kind of systems has been addressed by various researchers [1-4] to obtain maximal uncertainty bounds for preserving stability.

Similar to the case of stability robustness, the performance robustness problem of uncertain systems has also gained considerable attention in the last decade. In a large number of literatures, the robust performance problem deals with the computation of the worst case performance index when the system under consideration is subject to norm bounded uncertainties [5-7]. In contrast, here we are interested in determining parametric uncertainty bounds for the preservation of a

(C) IEE, 1996

IEE Proceedings online no. 19960807

Paper received 12th June 1996

The authors are with the Department of Electrical Engineering, National Taiwan University, Taipei, Taiwan 10617, Republic of China selected performance specification: the system $\mathrm{H}_{2}$ norm [8]. Through matrix nonsingularity analysis [4], we show that the desired bound can be expressed in terms of the structured singular values [9] of composite matrices formed by using known system and uncertainty structural information. The derived bound is necessary and sufficient and can be computed to quite good precision by using existing structured singular value analysis tools, such as [10].

As the structured singular value computation problems often become rather large when the system dimension and number of uncertain parameters are large, we propose an algorithm for reducing the matrix sizes involved in the calculation of uncertainty bounds. Theoretically, this amounts to utilising the structural information of uncertainties to express the original problem in a compact form. Numerically, with the matrix size reduction the computation speed will often be greatly improved for this well known NP-hard [11] analysis problem.

The notations adopted are as follows. Let $\|H\|_{2}$ be the $\mathrm{H}_{2}$ norm of the transfer function $H(s)$. $\pi$ represents the considered performance index. $\mu(M, \Omega)$ stands for the structured singular value of the matrix $M$ with respect to a set $\Omega$ of block diagonal uncertainty matrices of a fixed structure [9]. Sometimes the $\Omega$ part will be omitted for the sake of simplicity. The symbol col( $\cdot)$ denotes the column stacking operation [12], and $\bar{\sigma}$ is the maximal singular value. Finally, $\oplus$ refers to the Kronecker sum [12], and $I_{\delta}$ is the identity matrix of dimension $\delta$.

\section{Problem formulation}

Consider the following linear state-space model with uncertainties and constant output feedback:

$$
\begin{aligned}
& \dot{x}(t)=\left(A_{P 0}+\sum_{i=1}^{p} k_{i} A_{P i}\right) x(t)+\left(B_{P 0}+\sum_{i=1}^{p} k_{i} B_{P i}\right) u(t) \\
& y(t)=\left(C_{P 0}+\sum_{i=1}^{p} k_{i} C_{P i}\right) x(t)
\end{aligned}
$$$$
u(t)=w(t)-K y(t)
$$

where $x \in \mathbf{R}^{n}, u \in \mathbf{R}^{m}$, and $y \in \mathbf{R}^{l}$ are the state, input, and output vectors of the plant, respectively, $w \in \mathbf{R}^{m}$ is an exterior excitation, $\left\{A_{P 0}, B_{P 0}, C_{P 0}\right\}$ represent the nominal plant dynamics, and $K \in \mathbf{R}^{m \times l}$ is the unperturbed output feedback gain matrix. In eqn. $1, k_{i}, i=1$, 
$\ldots, p$, are real uncertain parameters, bounded by $\max _{i}\left|k_{i}\right|<r$. The matrices $\left\{A_{P_{i}}, B_{p_{i}}, C_{p_{i}}\right\}$ of suitable dimensions are the corresponding structural information matrices of $k_{i}$, and are assumed to be known. If $k_{i}$ does not appear in, for example, output matrix, $C_{P i}=$ 0 , etc. Substituting eqn. 3 into eqns. 1 and 2 , we see that the closed-loop system can be written as

$$
\begin{aligned}
\dot{x}(t)= & \left(A_{0}+\sum_{i=1}^{p} k_{i} A_{i}+\sum_{i, j=1}^{p} k_{i} k_{j} A_{i j}\right) x(t) \\
& +\left(B_{0}+\sum_{i=1}^{p} k_{i} B_{i}\right) w(t) \\
y(t)= & \left(C_{0}+\sum_{i=1}^{p} k_{i} C_{i}\right) x(t)
\end{aligned}
$$

or more compactly

$$
\begin{aligned}
& \dot{x}(t)=A(k) x(t)+B(k) w(t) \\
& y(t)=C(k) x(t)
\end{aligned}
$$

where $A_{0}=A_{P 0}-B_{P 0} K C_{P 0}, A_{i}=A_{P i}-B_{P 0} K C_{P i}-$ $B_{P i} K C_{P 0}, A_{i j}=-B_{P i} K C_{P j}, B_{0}=B_{P 0}, B_{i}=B_{P i}, C_{0}=C_{P 0}$, $C_{i}=C_{P i}$, and $k=\left[\begin{array}{lllll}k_{1} & k_{2} & \ldots & k_{p}\end{array}\right]^{T}$. For the sake of generality, it is assumed that some $A_{i}, A_{i j}, B_{i}$, and $C_{i}$ are nonzero. Also, it is assumed that $\left\{A_{0}, B_{0}, C_{0}\right\}$ is a minimal realisation.

To discuss the performance of a system, we must have stability first. Thus we assume further that the nominal system is asymptotically stable (i.e. eigenvalues of $A_{0}$ all lie in the open left half complex plane). Then we can use the method of [4] to find a range of $k$ within which eqn. 5 is asymptotically stable. The result is a necessary and sufficient condition, having the form

$$
\max _{i}\left|k_{i}\right|<1 / \mu\left(M_{s}\right)
$$

where $M_{s}$ is a matrix depending on $A_{0}, A_{i} \mathrm{~s}$, and $A_{i j} \mathrm{~s}$. Hereafter we shall assume that $r$ is equal to $1 / \mu\left(M_{s}\right)$, so that the discussion of performance robustness is meaningful.

The performance robustness problem we want to consider is as follows. Suppose that we have a performance index $\pi\{A(k), B(k), C(k)\}$ which is a continuous function of $k$, and we know its value for the nominal system: $\pi\left\{A_{0}, B_{0}, C_{0}\right\}=\pi_{0}>0$. Given a performance bound $\pi_{B}>\pi_{0}$, we want to find the largest range of $k$ within which $\pi\{A(k), B(k), C(k)\}<\pi_{B}$. Equivalently, we want to find

$r_{\pi} \triangleq \inf \left\{\max _{i}\left|k_{i}\right|: \pi\{A(k), B(k), C(k)\}=\pi_{B}, \max _{i}\left|k_{i}\right|<r\right\}$

Note that, due to the continuity of $\pi\{A(k), B(k), C(k)\}$, if there is no $k$ in the set $\left\{k\right.$ : $\left.\max _{i}\left|k_{i}\right|<r\right\}$ making $\pi\{A(k), B(k), C(k)\}=\pi_{B}$, all $k$ in the set are such that $\pi\{A(k), B(k), C(k)\}<\pi_{B}$. In this case, we say $r_{\pi}=r$. For the case in which $r_{\pi}$ can not be computed exactly, we shall try to find a lower bound for it.

\section{$3 \quad \mathrm{H}_{2}$ norm performance robustness}

In this Section, our discussion focuses on the system $\mathrm{H}_{2}$ norm performance.

The $H_{2}$ norm performance index represents the rootmean-square value of the output when the input $w(t)$ in eqn. 5 is driven by zero-mean white noises with unit covariance. For convenience, we consider the squared performance index $\pi\{A(k), B(k), C(k)\}=\| C(k)\left[s I_{n}-\right.$ $A(k)]^{-1} B(k) \|_{2}^{2}$, which can be expressed as $[8,12]$

$\left\|C(k)\left[s I_{n}-A(k)\right]^{-1} B(k)\right\|_{2}^{2}$

$$
=\operatorname{col}^{T}\left[C(k)^{T} C(k)\right][A(k) \oplus A(k)]^{-1} \operatorname{col}\left[-B(k) B(k)^{T}\right]
$$

Since the asymptotic stability of $A(k)$ guarantees [12] the nonsingularity of $A(k) \oplus A(k), \pi\{A(k), B(k), C(k)\}$ is a continuous function of $k$ in the region specified by eqn. 6 .

According to the standard problem formulation defined in Section 2, assume $\pi\left\{A_{0}, B_{0}, C_{0}\right\}=\pi_{0}>0$, and a $\pi_{B}>\pi_{0}$ is given. We must find the smallest $\max _{i}\left|k_{i}\right|$ making $\pi\{A(k), B(k), C(k)\}=\pi_{B}$. A Lemma is proved first.

Lemma 1: For all $\pi>\pi_{0}$, the matrix

$$
\left[\begin{array}{cc}
-\pi & \operatorname{col}\left[C_{0}^{T} C_{0}\right] \\
\operatorname{col}\left[B_{0} B_{0}^{T}\right] & A_{0} \oplus A_{0}
\end{array}\right]
$$

is nonsingular.

Proof: Since $\pi_{0}=\operatorname{col}^{T}\left[C_{0}^{T} C_{0}\right]\left(A_{0} \oplus A_{0}\right)^{-1} \operatorname{col}\left[-B_{0} B_{0}^{T}\right]$ and $\pi>\pi_{0}$, it follows immediately that

$$
\pi+\operatorname{col}^{T}\left[C_{0}^{T} C_{0}\right]\left(A_{0} \oplus A_{0}\right)^{-1} \operatorname{col}\left[B_{0} B_{0}^{T}\right] \neq 0
$$

Moreover, we have $\operatorname{det}\left(A_{0} \oplus A_{0}\right) \neq 0$ and

$$
\begin{aligned}
& \operatorname{det}\left[\begin{array}{cc}
-\pi & \operatorname{col}\left[C_{0}^{T} C_{0}\right] \\
\operatorname{col}\left[B_{0} B_{0}^{T}\right] & A_{0} \oplus A_{0}
\end{array}\right] \\
& =\operatorname{det}\left(A_{0} \oplus A_{0}\right) \\
& \quad \times \operatorname{det}\left\{-\pi-\operatorname{col}^{T}\left[C_{0}^{T} C_{0}\right]\left(A_{0} \oplus A_{0}\right)^{-1} \operatorname{col}\left[B_{0} B_{0}^{T}\right]\right\} \\
& \neq 0
\end{aligned}
$$

The conclusion holds.

To proceed, we need some more matrices defined for $i, j=1, \ldots, p$ and $\pi_{B}>\pi_{0}$,

$$
\begin{aligned}
& E_{i}=\left[\begin{array}{cc}
0 & \operatorname{col}^{T}\left[C_{0}^{T} C_{i}+C_{i}^{T} C_{0}\right] \\
\operatorname{col}\left[B_{0} B_{i}^{T}+B_{i} B_{0}^{T}\right] & A_{i} \oplus A_{i}
\end{array}\right] \\
& \cdot\left[\begin{array}{cc}
-\pi_{B} & \operatorname{col}^{T}\left[C_{0}^{T} C_{0}\right] \\
\operatorname{col}\left[B_{0} B_{0}^{T}\right] & A_{0} \oplus A_{0}
\end{array}\right]^{-1} \\
& E_{i j}=\left[\begin{array}{cc}
0 & \operatorname{col}^{T}\left[C_{i}^{T} C_{j}\right] \\
\operatorname{col}\left[B_{i} B_{j}^{T}\right] & A_{i j} \oplus A_{i j}
\end{array}\right] \\
& \cdot\left[\begin{array}{cc}
-\pi_{B} & \operatorname{col}^{T}\left[C_{0}^{T} C_{0}\right] \\
\operatorname{col}\left[B_{0} B_{0}^{T}\right] & A_{0} \oplus A_{0}
\end{array}\right]^{-1} \\
& E_{e}=\left[\begin{array}{c}
E_{1} \\
\vdots \\
E_{p}
\end{array}\right] \quad E_{e e}=\left[\begin{array}{ccc}
E_{11} & \cdots & E_{1 p} \\
\vdots & \ddots & \vdots \\
E_{p 1} & \cdots & E_{p p}
\end{array}\right] \\
& \bar{E}=\left[\begin{array}{cc}
E_{e} & E_{e e} \\
-I_{n^{2}+1} & 0
\end{array}\right] \\
& U_{i}=\left(e_{1} e_{i}^{T}+e_{i+1} e_{p+1}^{T}\right) \otimes I_{\left(n^{2}+1\right)} \\
& e_{j}=\text { the } j \text { th column of } I_{p+1}
\end{aligned}
$$

where $E_{i} \in \mathbf{R}^{\left(n^{2}+1\right) \times\left(n^{2}+1\right)}, \quad E_{i j} \in \mathbf{R}^{\left(n^{2}+1\right) \times\left(n^{2}+1\right)}$ $E_{e} \in \mathbf{R}^{p\left(n^{2}+1\right) \times\left(n^{2}+1\right)}, \quad E_{e e} \in \mathbf{R}^{p\left(n^{2}+1\right) \times p\left(n^{2}+1\right)}, \bar{E} \in$ $\mathbf{R}^{(p+1)\left(n^{2}+1\right) \times(p+1)\left(n^{2}+1\right)}$, and $U_{i} \in \mathbf{R}^{(p+1)\left(n^{2}+1\right) \times(p+1)\left(n^{2}+1\right)}$. Note that, by Lemma 1 , these matrices are well defined. Now we are in the position to present the first result.

Theorem 1: Suppose the nominal squared $H_{2}$ norm performance index of system eqn. 5 is equal to $\pi_{0}>0$. 
For a performance bound $\pi_{B}>\pi_{0}$

$$
r_{\pi}=\min \left\{1 / \mu\left(M_{s}\right), 1 / \mu\left(M_{2}, \Omega_{2}\right)\right\}
$$

where

$$
\begin{aligned}
& M_{2}=\left[\begin{array}{c}
U_{1} \overline{\bar{E}} \\
\vdots \\
U_{p} \bar{E}
\end{array}\right]\left[I_{(p+1)\left(n^{2}+1\right)} \cdots I_{(p+1)\left(n^{2}+1\right)}\right] \\
& \in \mathbf{R}^{\left(p^{2}+p\right)\left(n^{2}+1\right) \times\left(p^{2}+p\right)\left(n^{2}+1\right)}
\end{aligned}
$$

and

$$
\begin{aligned}
\Omega_{2}=\{\Delta & =\operatorname{diag}\left[k_{1} I_{(p+1)\left(n^{2}+1\right)}, \ldots, k_{p} I_{(p+1)\left(n^{2}+1\right)}\right]: \\
& \left.k_{i} \in \mathbf{R}, i=1, \ldots, p\right\}
\end{aligned}
$$

Proof: To find the smallest $\max _{i}\left|k_{i}\right|$ such that $\pi\{A(k)$, $B(k), C(k)\}=\pi_{B}$, let

$\pi_{B}=\operatorname{col}^{T}\left[C(k)^{T} C(k)\right][A(k) \oplus A(k)]^{-1} \operatorname{col}\left[-B(k) B(k)^{T}\right]$

Simple manipulation yields

$$
\begin{aligned}
& 1+\frac{1}{\pi_{B}} \operatorname{col}^{T}\left[C(k)^{T} C(k)\right][A(k) \oplus A(k)]^{-1} \\
& \times \operatorname{col}\left[B(k) B(k)^{T}\right]=0
\end{aligned}
$$

Using the identity $\operatorname{det}\left(I_{\alpha}+X Y\right)=\operatorname{det}\left(I_{\beta}+Y X\right)$ for all $X \in \mathbf{R}^{\alpha \times \beta}$ and $Y \in \mathbf{R}^{\beta \times \alpha}$, we get

$$
\begin{gathered}
\operatorname{det}\left\{I_{n}^{2}+\frac{1}{\pi_{B}}[A(k) \oplus A(k)]^{-1} \operatorname{col}\left[B(k) B(k)^{T}\right]\right. \\
\left.\quad \times \operatorname{col}^{T}\left[C(k)^{T} C(k)\right]\right\}=0
\end{gathered}
$$

Multiplying both sides by $\operatorname{det}[A(k) \oplus A(k)] \neq 0$ results in

$$
\begin{aligned}
\operatorname{det}\{[ & {[A(k) \oplus A(k)]-\operatorname{col}\left[B(k) B(k)^{T}\right]\left(-\frac{1}{\pi_{B}}\right) } \\
& \left.\times \operatorname{col}^{T}\left[C(k)^{T} C(k)\right]\right\}=0
\end{aligned}
$$

With some matrix elementary operations, it is easy to show that the above is equivalent to

$$
\operatorname{det}\left[\begin{array}{cc}
-\pi_{B} & \operatorname{col}^{T}\left[C(k)^{T} C(k)\right] \\
\operatorname{col}\left[B(k) B(k)^{T}\right] & A(k) \oplus A(k)
\end{array}\right]=0
$$

which in detailed form is

$$
\begin{aligned}
& \operatorname{det}\left\{\begin{array}{cc}
-\pi_{B} & \operatorname{col}^{T}\left[C_{0}^{T} C_{0}\right] \\
\operatorname{col}\left[B_{0} B_{0}^{T}\right] & A_{0} \oplus A_{0}
\end{array}\right] \\
& \quad+\sum_{i=1}^{p} k_{i}\left[\begin{array}{cc}
0 & \operatorname{col}^{T}\left[C_{0}^{T} C_{i}+C_{i}^{T} C_{0}\right] \\
\operatorname{col}\left[B_{0} B_{i}^{T}+B_{i} B_{0}^{T}\right] & A_{i} \oplus A_{i}
\end{array}\right] \\
& \left.\quad+\sum_{i, j=1}^{p} k_{i} k_{j}\left[\begin{array}{cc}
0 & \operatorname{col}^{T}\left[C_{i}^{T} C_{j}\right] \\
\operatorname{col}\left[B_{i} B_{j}^{T}\right] & A_{i j} \oplus A_{i j}
\end{array}\right]\right\}=0
\end{aligned}
$$

Since the first block matrix is nonsingular by Lemma 1 , we may also rewrite the above as

$$
\operatorname{det}\left\{I_{n^{2}+1}+\sum_{i=1}^{p} k_{i} E_{i}+\sum_{i, j=1}^{p} k_{i} k_{j} E_{i j}\right\}=0
$$

which falls right into the form of the nonsingularity analysis problem discussed in [4]. Thus the result follows by straightforward application of the method developed in [4].

It is worth mentioning that the above result is a necessary and sufficient condition theoretically. For the cases in which the corresponding structured singular values can be computed exactly, such as the single uncertain parameter case, $r_{\pi}$ given by Theorem 1 is the largest bound to ensure $\mathrm{H}_{2}$ norm performance robust- ness in the sense defined in this paper. Hence no conservativeness exists. If the corresponding structured singular values cannot be evaluated precisely, numerical methods may be adopted to compute reasonably good bounds for $r_{\pi}$. Furthermore, the result in Section 4 may help reduce the problem sizes.

\section{Size reduction of the computation problem}

In Section 3, the performance robustness problem results in structured singular value computation problems of the form: to decide $\mu(M, \Omega)$ with $M=[W W \ldots$ $W$ for some matrix $W$, and $\Omega$ a set of diagonal matrices $\Delta=\operatorname{diag}\left[k_{1} I, k_{2} I, \ldots, k_{p} I\right]$. Actually this is the same form of computation problem resulted from the robust stability analysis problem [4]. When the system in discussion has a large state number $n$ and an uncertain parameter number $p$, the sizes of $M$ and $\Delta$ will become quite large. Although, up to now, many algorithms have been developed for computing structured singular values to a good precision, the required computation time grows rapidly with the problem size. Thus it is beneficial to do problem size reduction whenever possible. Before we present our result in this regard, a generalisation is introduced first, so that the robust nonsingularity analysis problem involving fractional type uncertainties [13] can also be discussed. Below, we shall consider problems in which the matrix $M$ does not have repeated blocks $W \mathrm{~s}$, but has different blocks $W_{a}, W_{b}$, etc. The set $\Omega$ is changed accordingly. To be simultaneously general and neat, we consider the computation of $\mu(M, \Omega)$ with

$$
M=\left[W_{a} W_{b}\right] \in \mathbf{R}^{q \times q}
$$

and

$$
\Omega=\left\{\Delta=\operatorname{diag}\left[k_{1} I_{q_{a}}, k_{2} I_{q_{b}}\right]\right\}
$$

where $W_{a} \in \mathbf{R}^{q \times q_{a}}$ has rank $\rho_{a}, W_{b} \in \mathbf{R}^{q \times q_{b}}$ has rank $\rho_{b}$, and $q_{a}+q_{b}=q$. It will be clear from the following discussion how to deal with cases in which there are more than two $W$ s. Now, we show that the problem size can be cut down if $\rho_{i}<q_{i}$ for any $i=a, b$. Again, for the sake of generality, we assume $\rho_{i}<q_{i}$ for both $i=a, b$. Let the singular value decomposition of $W_{i}, i=a, b$, respectively, be $U_{i} \Sigma_{i} V_{i}^{T}$ where $U_{i} \in \mathbf{R}^{q \times q}, V_{i} \in \mathbf{R}^{q i \times q_{i}}$ are unitary, and $\Sigma_{i} \in \mathbf{R}^{g \times q i}$ has positive singular values in the first $\rho_{i}$ diagonal positions and zeros elsewhere. So we represent each $W_{i}$ in the partitioned form

$$
W_{i}=\left[\begin{array}{ll}
U_{i 1} & U_{i 2}
\end{array}\right]\left[\begin{array}{cc}
\hat{\Sigma}_{i} & 0 \\
0 & 0
\end{array}\right] V_{i}^{T}
$$

where $\hat{\Sigma}_{i} \in \mathbf{R}^{\rho i x p i}$ is the upper right part of $\Sigma_{i}$ and $\left[\begin{array}{ll}U_{i 1} & U_{i 2}\end{array}\right]=U_{i}$ partitioned accordingly. Since $V_{i}$ is unitary, we have

$$
W_{i} V_{i}=U_{i} \Sigma_{i}=\left[\begin{array}{ll}
U_{i 1} \hat{\Sigma}_{i} & 0
\end{array}\right]
$$

With $V=\operatorname{diag}\left[V_{a}, V_{b}\right]$, we can modify the key equation $\operatorname{det}\left(I_{q}+M \Delta\right)=0$ in the structured singular value computation problem as

$$
\operatorname{det}\left(I_{q}+V^{T} M \Delta V\right)=\operatorname{det}\left(I_{q}+V^{T} M V \Delta\right)=0
$$

Thus we have

$$
\begin{aligned}
& \operatorname{det}\left\{I_{q}+\left[V^{T} U_{a 1} \hat{\Sigma}_{a} \quad 0 \quad V^{T} U_{b 1} \hat{\Sigma}_{b} \quad 0\right]\left[\begin{array}{cc}
k_{1} I_{q_{a}} & 0 \\
0 & k_{2} I_{q_{b}}
\end{array}\right]\right\} \\
& =0
\end{aligned}
$$

For $i=a, b$, let $\tilde{W}_{i}$ denote the submatrix of $V^{T} U_{i 1} \hat{\Sigma}_{i}$ formed by deleting the $\left(\rho_{a}+1\right)$ st to the $q_{a}$ th and the 
$\left(q_{a}+\rho_{b}+1\right)$ st to the $\left(q_{a}+q_{b}\right)$ th rows. Also, let $\tilde{\Delta}=$ $\operatorname{diag}\left[k_{1} I_{\rho_{a}}, k_{2} I_{\rho_{b}}\right]$. Then eqn. 18 is equivalent to

$$
\operatorname{det}\left\{I_{\rho_{a}+\rho_{b}}+\left[\tilde{W}_{a} \tilde{W}_{b}\right] \tilde{\Delta}\right\}=0
$$

If we define $\tilde{M}$ to be $\left[\begin{array}{ll}\tilde{W}_{a} & \tilde{W}_{b}\end{array}\right]$ and $\tilde{\Omega}$ to be the set composed of all diagonal matrices of the form $\tilde{\Delta}$, we can summarise the above result in the following theorem.

Theorem 2: $\mu(M, \Omega)=\mu(\tilde{M}, \tilde{\Omega})$, and the size of the new problem is smaller than that of the original one by $q-\left(\rho_{a}+\rho_{b}\right)$.

It is worth mentioning that in some cases the above size reduction step may be repeated. For example, let $q_{a}=3, q_{b}=1$, and

$$
M=\left[\begin{array}{cccc}
2 & 1 & -3 & -5 \\
1 & 1 & -2 & 4 \\
0 & 1 & -1 & 3 \\
5 & 0 & -5 & 2
\end{array}\right]
$$

$\rho_{a}=2$ and $\rho_{b}=1$, so the problem size may be reduced from 4 to 3 , with $\tilde{q}_{a}=2$ and $\tilde{q}_{b}=1$. However, it can be checked that $\tilde{W}_{a}$ has only rank one, therefore the problem size can be further reduced to 2 by repeating the procedure.

Moreover, note that, because $\Delta$ is diagonal, $\operatorname{det}(I+M \Delta)=\operatorname{det}\left(I+\Delta^{T} M^{T}\right)=\operatorname{det}\left(I+M^{T} \Delta\right)$

Hence we can also consider the possibility of problem size reduction for $\mu\left(M^{T}, \Omega\right)$ instead of $\mu(M, \Omega)$, provided the latter is irreducible in size by the above procedure. For example, let $q_{a}=3, q_{b}=1$, and

$$
M=\left[\begin{array}{cccc}
1 & -3 & 2 & -1 \\
3 & -1 & -2 & 3 \\
2 & -2 & 0 & 1 \\
5 & 4 & -9 & -2
\end{array}\right]
$$

It is interesting to note that the problem size can be cut down from 4 to 3 by using the standard procedure, and further cut down to 2 by using the same procedure to $\mu\left(\tilde{M}^{T}, \tilde{\Omega}\right)$.

As a final remark, we mention that, in the structured singular value computation problems generated in this paper, the matrix $M$ does have repeated $W$ blocks, which are related to the system and uncertain parameter structural matrices. If $W$ is rank deficient due to, for example, the sparsity of those matrices, the size of $M$ may be reduced quite significantly, because of the multiplicity of $W$. Thus, the overall structural information are utilised to express the original problem in a more compact form.

\section{Example}

Consider the following uncertain system with output feedback:

$$
\begin{aligned}
& \dot{x}=\left[\begin{array}{cc}
-1+k_{1}+k_{2} & -1 \\
2 k_{1} & 2-2 k_{2}
\end{array}\right] x+\left[\begin{array}{c}
1-k_{2} \\
-3
\end{array}\right] u \\
& y=\left[\begin{array}{ll}
0 & 1.25-k_{1}
\end{array}\right] x \\
& u=w+3 y
\end{aligned}
$$

The closed-loop system is described by

$$
\begin{aligned}
& \dot{x}= {\left[\begin{array}{cc}
-1+k_{1}+k_{2} & 2.75-3 k_{1}-3.75 k_{2}+3 k_{1} k_{2} \\
2 k_{1} & -9.25+9 k_{1}-2 k_{2}
\end{array}\right] x } \\
&+\left[\begin{array}{c}
1-k_{2} \\
-3
\end{array}\right] w \\
& y= {\left[\begin{array}{ll}
0 & 1.25-k_{1}
\end{array}\right] x } \\
& 512
\end{aligned}
$$

When $k_{1}=k_{2}=0$, the system eigenvalues are -1 and -9.25 , which imply nominal asymptotic stability. For the closed-loop system, the robust matrix nonsingularity analysis method of [4] gives the robust stability region $\max _{i}\left|k_{i}\right|<1 / \mu\left(M_{s}\right)=0.4847$. Thus, we assume that the uncertain parameters $k_{1}$ and $k_{2}$ are confined in this region, and examine the $\mathrm{H}_{2}$ norm performance robustness of the system. Based on the formula given in Section 3, $\pi_{0}$, the squared $H_{2}$ norm performance index of the nominal system, can be calculated to be 0.7601 . If we specify $\pi_{B}=2$, Theorem 1 gives $r_{\pi}=$ 0.4839 .

Regarding the usefulness of the result developed in Section 4, we note that, to determine the above squared $\mathrm{H}_{2}$ norm performance robustness region directly, we must compute the structured singular value of a $30 \times$ 30 matrix with respect to uncertainty matrices of the form $\Delta=\operatorname{diag}\left[k_{1} I_{15}, k_{2} I_{15}\right]$. The computation is accomplished by using the software [10], and the cumulative floating point operation count is 31475897 flops. However, with the procedure of Section 4 , the matrix size involved becomes only $14 \times 14$, and the uncertainty matrices are of the form $\tilde{\Delta}=\operatorname{diag}\left[k_{1} I_{7}, k_{2} I_{7}\right]$. When the same software [10] is used to do the computation task, only 4038126 flops are needed, including the operations required to make the singular value decompositions. This is a reduction of $87 \%$.

\section{Conclusion}

In this paper, we propose an algorithm to find the uncertain parameter bound for linear state-space models which guarantees the system $\mathrm{H}_{2}$ norm performance index is under a prespecified value. The result is expressed in terms of structured singular values of matrices composed of system and uncertainty structural information. To improve computation efficiency, a method is developed to reduce the size of composite matrices. As there are many more robustness analysis problems that can be transformed into the structured singular value computation problem considered here [4, 13], it is believed that the results of this paper not only add one more element into the framework but also contribute a computation aid to all these problems.

\section{Acknowledgments}

This research is supported by the National Science Council of the Republic of China under Grant NSC 84-2213-E002-082 and NSC 85-2213-E002-039.

\section{References}

1 FU, M, and BARMISH, B.R.: 'Maximal unidirectional perturbation bounds for stability of polynomials and matrices', Syst. Control Lett., 1988, 11, pp. 173-179

2 TESI, A., and VICINO, A.: 'Robust stability of state-space models with structured uncertainties', IEEE Trins., 1990, AC-35, (2), pp. 191-195

3 SU, J.H., and FONG, I-K.: 'Robust stability analysis of linear continuous/discrete-time systems with output feedback controllers', IEEE Trans., 1993, AC-38, (7), pp. 1154-1158

4 TSENG, C.L., FONG, I-K., and SU, J.H.: 'Analysis and applications of robust non-singularity problem using the structured singular value', IEEE Trans., 1994, AC-39, (10), pp. 2118-2122

5 FRIEDMAN, J.H., KABAMBA, P.T., and KHARGONECHAR, P.P.: "Worst-case and average $\mathrm{H}_{2}$ performance analysis against real constant parametric uncertainty". Proceedings of 1994 ACC, Baltimore, pp. 2406-2410 
6 KHAMMASH, M.H.: 'Robust performance bounds for systems with time-varying uncertainty'. Proceedings of 33rd CDC, Lake Buena Vista, 1994, pp. 28-33

7 ZHOU, K., GLOVER, K., BODENHEIMER, B., and DOYLE, J.C.: 'Mixed $H_{2}$ and $H_{\infty}$ performance objectives, I: robust performance analysis', IEEE Trans., 1994, AC-39, (8), pp. 1564 1574

8 BOYD, S.P., and BARRATT, C.H.: 'Linear controller design: limit of performance' (Prentice-Hall, 1991)

9 DOYLE, J.C.: 'Analysis of feedback systems with structured uncertainties', IEE Proc. D, 1982, 129, pp. 242-250
10 BALAS, G.J., DOYLE, J.C., GLOVER, K, PACKARD, A. and SMITH, R.: ' $\mu$-analysis and synthesis toolbox-user's guide' (Musyn Inc. and The MathWorks Inc., Natick, 1991)

11 POLJAK, S., and ROHN, J.: 'Checking robust nonsingularity is NP hard', Math. Contr. Sig. Syst., 1993, 6, pp. 1-9

12 WEINMANN, A.: 'Uncertain models and robust control' (Springer-Verlag, New York, 1991)

13 TSENG, C.L., and FONG, I-K.: 'Robust nonsingularity analysis using linear fractional transformation and its application to stability analysis'. Proceedings of 33rd CDC, Lake Buena Vista, 1994, pp. 589-590 